\title{
The neutron EDM and bounds on rare decays of eta and eta- prime mesons
}

\author{
Alexey S. Zhevlakov ${ }^{1, *}$, Thomas Gutsche ${ }^{2}$, and Valery E. Lyubovitskij ${ }^{1,2,3}$ \\ ${ }^{1}$ Department of Physics, Tomsk State University, 634050 Tomsk, Russia \\ ${ }^{2}$ Institut für Theoretische Physik, Universität Tübingen, Kepler Center for Astro and Particle Physics, \\ Auf der Morgenstelle 14, D-72076 Tübingen, Germany \\ ${ }^{3}$ Departamento de Física y Centro Científico Tecnológico de Valparaíso-CCTVal, Universidad Técnica \\ Federico Santa María, Casilla 110-V, Valparaíso, Chile
}

\begin{abstract}
We complete our derivation of upper limits on the $\mathrm{CP}$ violating $\eta \pi \pi$ and $\eta^{\prime} \pi \pi$ couplings from an analysis of their two-loop contribution to the neutron electric dipole moment (nEDM). As a result we obtain numerical upper limits for the $\eta \pi \pi$ and $\eta^{\prime} \pi \pi$ branchings and couplings which are important for planned experiments at JLab. We also derive upper limits on the CP violating $\bar{\theta}$ parameter of QCD $\bar{\theta}<2.8 \times 10^{-10}$.
\end{abstract}

\section{Introduction}

The study of CP-violation in particle physics reaches back over more than 60 years. In particular, the experimental confirmation of CP-violation in the weak interaction sector is well established, as for example for $\mathrm{K}$ and $\mathrm{B}$ meson physics, and the phenomena are well-explained in the theoretical framework of the Standard Model (SM). An important topic in the study of $\mathrm{CP}$-violating physics is the investigation of an electric dipole moment (EDM) of particles, as the electron, neutron or others [1]. The SM prediction for the neutron EDM (nEDM) is several orders lower than existing experimental limits. This also gives the opportunity to search for additional CP-violating sources which could be based on New Physics or are based on $\mathrm{CP}$-violation in the strong interaction sector.

In quantum chromodynamics (QCD) an nEDM can be induced due to the addition of a $\mathrm{CP}$-violating $\theta$-term into the action with

$$
\Delta \mathcal{L}=\theta \frac{g_{s}^{2}}{32 \pi^{2}} G_{\mu \nu}^{a} \tilde{G}^{a \mu \nu}
$$

where $g_{s}$ is the QCD coupling constant, and $G_{\mu \nu}^{a}$ and $\tilde{G}^{a \mu \nu}=\frac{1}{2} \epsilon^{\mu \nu \alpha \beta} G_{\alpha \beta}^{a}$ are the usual stress tensor of gluon fields and its dual. The theta term breaks the P- and T-parity invariance and plays an important role in the description of strong interaction and its properties, in particular, the QCD vacuum at low energy. It gives a solution of the $U(1)_{A}$ problem of the $\eta^{\prime}$ meson mass (see, e.g. Refs. [6, 7]).

The $\theta$ - term plays a role for the nEDM due to the generation of a CP-violating term in the strong interaction beween baryon and pseudoscalar fields[8]. A generation of an nEDM is

\footnotetext{
*e-mail: zhevlakov@phys.tsu.ru
} 
also possible by taking into account the CP-violating transitions of $\eta$ and $\eta^{\prime}$ to a pair of pions. The relevant couplings are also connected to the $\theta$ - parameter of CP-violation [9] as:

$$
f_{\eta \pi \pi}^{\bar{\theta}}=-\frac{1}{\sqrt{3}} \frac{\bar{\theta} M_{\pi}^{2} R}{F_{\pi} M_{\eta}(1+R)^{2}}, \quad f_{\eta^{\prime} \pi \pi}^{\bar{\theta}}=\sqrt{2} f_{\eta \pi \pi}^{\bar{\theta}} \frac{M_{\eta}}{M_{\eta^{\prime}}},
$$

where $\bar{\theta}$ is the QCD vacuum angle, $R=m_{u} / m_{d}$ is ratio of masses of the $u$ and $d$ current quarks, $F_{\pi}=92.4 \mathrm{MeV}$ is the pion decay constant, and $M_{\pi}=139.57 \mathrm{MeV}$ is the charged pion mass. Existing experiments give only upper limits for these decay widths [10]. Branchings of these CP-violating decays related to data of nEDM result in values which are orders of magnitude lower than the ones obtained from the direct experiment. Nevertheless, existing and future experimental programs for studying such rare CP-violating $\eta$ and $\eta^{\prime}$ decays have the aim to increase the sensitivity and search for possible sources related to new physics.

In addition, the study of model parameters of $\mathrm{CP}$-violation gives a chance to investigate $\mathrm{CP}$-violation at the next level — nuclear, atomic or molecular. Measurements at this new level can also give information and bounds on CP-violating parameters or the possibility to search for new physics beyond the SM. Future experiments will improve the sensitivity by one order of magnitude to $\sim 10^{-27}-10^{-28} e \cdot \mathrm{cm}$ using a variety of different technical approaches and experimental modifications.

To get an estimate for a possible nEDM by taking into account the $\eta$ and $\eta^{\prime}$ into two pions transition processes two-loop Feynman diagrams have to be evaluated. For simplicity we use a pseudoscalar (PS) approach with the non-derivative pion-nucleon coupling $g_{\pi N N} i \gamma_{5}$. We focus on the determination of the $C P$ violating couplings $\eta \pi \pi$ and $\eta^{\prime} \pi \pi$ from the bounds on the nEDM. Our formalism is based on a phenomenological Lagrangian describing the interaction of nucleons with pseudoscalar mesons (pions and $\eta\left(\eta^{\prime}\right)$ mesons). The interaction of charged particles with photons has been introduced via minimal and non-minimal substitution.

\section{Framework}

We use a phenomenological Lagrangian $\mathcal{L}_{\text {eff }}$ formulated in terms of hadronic degrees of freedom (nucleons $N=(p, n)$, pions $\pi=\left(\pi^{ \pm}, \pi^{0}\right), H=\left(\eta, \eta^{\prime}\right)$ mesons) and photons $A_{\mu}$. It separates into a free $\mathcal{L}_{0}$ and an interaction part $\mathcal{L}_{\text {int }}$ with

$$
\mathcal{L}_{\text {eff }}=\mathcal{L}_{0}+\mathcal{L}_{\text {int }} .
$$

$\mathcal{L}_{0}$ includes the usual free terms of nucleons, mesons, and photons

$$
\mathcal{L}_{0}=\bar{N}\left(i \not \partial-M_{N}\right) N+\frac{1}{2} \vec{\pi}\left(\square-M_{\pi}^{2}\right) \vec{\pi}+\frac{1}{2} H\left(\square-M_{H}^{2}\right) H-\frac{1}{4} F_{\mu \nu} F^{\mu \nu},
$$

where $\square=-\partial_{\mu} \partial^{\mu}, F_{\mu \nu}=\partial_{\mu} A_{\nu}-\partial_{v} A_{\mu}$ is the stress tensor of the electromagnetic field, $M_{N}, M_{\pi}$, and $M_{H}$ are the masses of nucleons, pions, and $\eta\left(\eta^{\prime}\right)$ mesons, respectively. The interaction Lagrangian $\mathcal{L}_{\text {int }}$ is given by a sum of two parts. The first part contains the strong interaction terms, which describe the CP-even couplings of nucleons with pions $\mathcal{L}_{\pi N N}$ and $\eta\left(\eta^{\prime}\right)$ mesons $\mathcal{L}_{H N N}$ and the CP-violating $\eta\left(\eta^{\prime}\right) \pi \pi$ coupling $\mathcal{L}_{H \pi \pi}^{\mathrm{CP}}$. The second part includes the electromagnetic interaction terms, describing the coupling of charged pions and nucleons 
with the photon $\left(\mathcal{L}_{\gamma N N}\right.$ and $\mathcal{L}_{\gamma \pi \pi}$, respectively):

$$
\begin{aligned}
\mathcal{L}_{\text {int }} & =\mathcal{L}_{\pi N N}+\mathcal{L}_{\eta\left(\eta^{\prime}\right) N N}+\mathcal{L}_{\eta\left(\eta^{\prime}\right) \pi \pi}^{\mathrm{CP}}+\mathcal{L}_{\gamma N N}+\mathcal{L}_{\gamma \pi \pi}, \\
\mathcal{L}_{\pi N N} & =g_{\pi N N} \bar{N} i \gamma_{5} \vec{\pi} \vec{\tau} N, \\
\mathcal{L}_{H N N} & =g_{H N N} H \bar{N} i \gamma_{5} N, \\
\mathcal{L}_{H \pi \pi}^{\mathrm{CP}} & =f_{H \pi \pi} M_{H} H \vec{\pi}^{2}, \\
\mathcal{L}_{\gamma N N} & =e A_{\mu} \bar{N}\left(\gamma^{\mu} Q_{N}+\frac{i \sigma^{\mu v} q_{v}}{2 M_{N}} k_{N}\right) N, \\
\mathcal{L}_{\gamma \pi \pi} & =e A_{\mu}\left(\pi^{-} i \partial^{\mu} \pi^{+}-\pi^{+} i \partial^{\mu} \pi^{-}\right)+e^{2} A_{\mu} A^{\mu} \pi^{+} \pi^{-},
\end{aligned}
$$

where $g_{\pi N N}=\frac{g_{A}}{F_{\pi}} M_{N}, g_{A}=1.275$ is the nucleon axial charge, $F_{\pi}=92.4 \mathrm{MeV}$ is the pion decay constant, $g_{H N N}$ and $f_{H \pi \pi}$ are corresponding CP-even and CP-odd couplings between pions and $\eta\left(\eta^{\prime}\right), \gamma^{\mu}$ and $\gamma^{5}$ are the Dirac matrices, and $\sigma^{\mu \nu}=\frac{i}{2}\left[\gamma^{\mu}, \gamma^{\nu}\right]$. The values of $g_{\eta N N}$ and $g_{\eta^{\prime} N N}$ are taken from Ref. [14]: $g_{\eta N N}=g_{\eta^{\prime} N N}=0.9$. Note that in the case of nucleons we include both minimal and nonminimal electromagnetic couplings. Here $Q_{N}=\operatorname{diag}(1,0)$ and $k_{N}=\operatorname{diag}\left(k_{p}, k_{n}\right)$ are the diagonal matrices of nucleon charges and anomalous magnetic moments, respectively, where $k_{p}=1.793$ and $k_{n}=-1.913$.

The pseudoscalar (PS) nucleon-meson coupling is equivalent to the more commonly used pseudovector (PV) theory (see details in [11-13]). The PS approach is convenient for the calculation of the nEDM taking into account two loop Feynman diagrams with the CP-violating $\eta\left(\eta^{\prime}\right) \rightarrow \pi \pi$ transition. Results for the relevant diagrams (Fig.1 and Fig.2.) do not have logarithmic divergences as in the PV case. Additional terms in the PV approach will be proportional to

$$
\sim \frac{1}{2 F_{\pi}^{2}}\left(g_{A}-1\right) \frac{i}{4 \pi^{2}} \Gamma(\epsilon)\left(\frac{4 \pi \mu^{2}}{M_{\pi}^{2}}\right)^{\epsilon}
$$

where $\mu$ is the parameter of dimension regularization (dimension $d=4-2 \epsilon$ ) and this term should be renormalized by introducing a low energy counter term in the PV theory.

\section{Neutron EDM}

The diagrams which describe a nEDM contribution due to a CP-violating transition of $\eta$ and $\eta^{\prime}$ mesons into two pions are shown in Fig.1 and Fig.2. The matrix element corresponding to the diagrams of Figs. 1 and 2 or the electromagnetic vertex function of the neutron is expanded in terms of four relativistic form factors $F_{E}$ (electric), $F_{M}$ (magnetic), $F_{D}$ (electric dipole) and $F_{A}$ (anapole) as

$$
\begin{aligned}
M_{\mathrm{inv}} & =\bar{u}_{N}\left(p_{2}\right) \Gamma^{\mu}\left(p_{1}, p_{2}\right) u_{N}\left(p_{1}\right), \quad \Gamma^{\mu}\left(p_{1}, p_{2}\right)=\gamma^{\mu} F_{E}\left(q^{2}\right)+\frac{i}{2 M_{N}} \sigma^{\mu v} q_{v} F_{M}\left(q^{2}\right) \\
& +\frac{1}{2 M_{N}} \sigma^{\mu v} q_{v} \gamma^{5} F_{D}\left(q^{2}\right)+\frac{1}{M_{N}^{2}}\left(\gamma^{\mu} q^{2}-2 M_{N} q^{\mu}\right) \gamma^{5} F_{A}\left(q^{2}\right)
\end{aligned}
$$

where $p_{1}$ and $p_{2}$ are the momenta of the initial and final neutron states, $q^{2}=\left(p_{2}-p_{1}\right)^{2}$ is the transfer momentum squared. The nEDM is defined as $d_{n}^{E}=-F_{D}(0) /\left(2 M_{N}\right)$.

The generic contribution of these diagrams to the $\mathrm{nEDM}$ is written as:

$$
-\bar{u}_{N}\left(p_{2}\right) d_{N}^{i} \sigma^{\mu v} q_{v} \gamma^{5} u_{N}\left(p_{1}\right)+\ldots=f_{H \pi \pi} g_{H N N} g_{\pi N N}^{2} M_{H}\left\{I_{\text {loop }}^{1}+\frac{k_{N}}{2 M_{N}} I_{\text {loop }}^{2}\right\},
$$

where $I_{\text {loop }}^{1}$ and $I_{\text {loop }}^{2}$ is sum of two Feynman diagrams. These diagrams provide an nEDM by interaction of the external electromagnetic field with minimal and non-minimal coupling to the baryon and pion field, respectively and are shown in Figs. 1 and 2. Details of the calculation of these two -oop Feynman diagrams (Fig.1 and Fig.2) can be found in [4, 5]. 


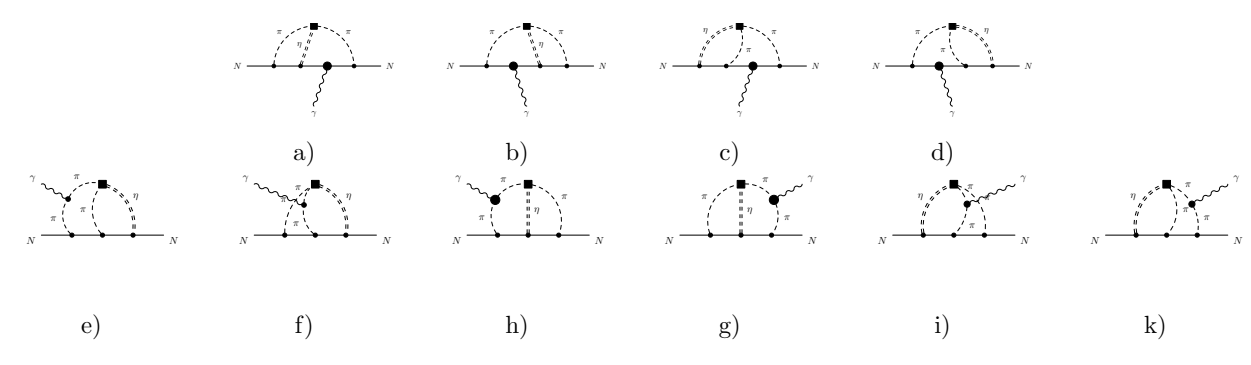

Figure 1. Diagrams contributing to the nEDM which are induced by the minimal electromagnetic couplings of proton and charged pions. The solid square denotes the CP-violating $\eta \pi^{+} \pi^{-}$vertex.

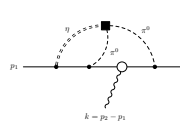

a)

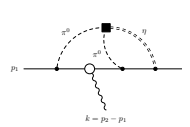

b )

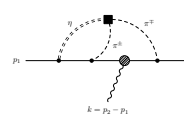

g)

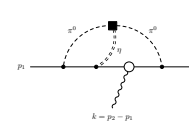

c)

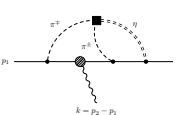

h)

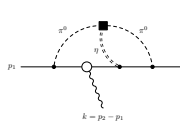

d )

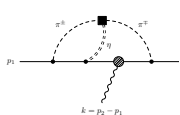

i)

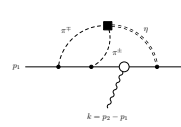

e)

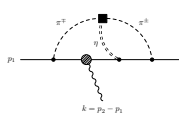

k)

Figure 2. Diagrams contributing to the nEDM which are induced by the nonminimal electromagnetic couplings (anomalous magnetic moments) of nucleons. The solid square denotes the CP-violating $\eta\left(\eta^{\prime}\right) \pi \pi$ vertex. Empty and shaded circles correspond to the nonminimal electromagnetic couplings of neutron and proton, respectively.

\section{Results and discussion}

Our numerical result for the nEDM induced by the CP violating $\eta\left(\eta^{\prime}\right) \pi \pi$ couplings and the anomalous magnetic moments of nucleons [5] is

$$
d_{n}^{E, k} \simeq\left(-0.14 f_{\eta \pi \pi}+-0.22 f_{\eta^{\prime} \pi \pi}\right) \times 10^{-16} \mathrm{e} \cdot \mathrm{cm} .
$$

The full result including both minimal and nonminimal electromagnetic couplings of nucleons can easily be computed by taking into account our previous results of Ref. [4] restricted to the case of minimal coupling:

$$
d_{n}^{E} \simeq\left(6.62 f_{\eta \pi \pi}+7.64 f_{\eta^{\prime} \pi \pi}\right) \times 10^{-16} \mathrm{e} \cdot \mathrm{cm} .
$$

The bounds for the branching ratios of the rare decays $\Gamma_{\eta \pi \pi}$ and $\Gamma_{\eta^{\prime} \pi \pi}$ are strongly suppressed when compared to existing data [10]

$$
\begin{array}{ll}
\operatorname{Br}\left(\eta \rightarrow \pi^{+} \pi^{-}\right)<5.54 \times 10^{-17}, & \operatorname{Br}\left(\eta^{\prime} \rightarrow \pi^{+} \pi^{-}\right)<5.33 \times 10^{-19}, \\
\operatorname{Br}\left(\eta \rightarrow \pi^{0} \pi^{0}\right)<2.27 \times 10^{-17}, & \operatorname{Br}\left(\eta^{\prime} \rightarrow \pi^{0} \pi^{0}\right)<2.17 \times 10^{-19} .
\end{array}
$$

The CP-violating $\eta \pi \pi$ and $\eta^{\prime} \pi \pi$ couplings are estimated using Eq. (9) and limits on the nEDM [16]:

$$
\left|f_{\eta \pi \pi}\left(M_{\eta}^{2}\right)\right|<4.4 \times 10^{-11}, \quad\left|f_{\eta^{\prime} \pi \pi}\left(M_{\eta^{\prime}}^{2}\right)\right|<3.8 \times 10^{-11} .
$$


We can extract $\bar{\theta}$ from the experimental limit on the nEDM [16] substituting the QCD relations between $f_{\eta\left(\eta^{\prime}\right) \pi \pi}^{\bar{\theta}}$ and $\bar{\theta}(2)$ into the expression for the nEDM (9). For the quark mass ratios taken from ChPT and LQCD we get:

$$
\begin{aligned}
& d_{n}^{E} \simeq\left(0.65 \times 10^{-16}\right) \cdot \bar{\theta} \mathrm{e} \cdot \mathrm{cm}(\mathrm{ChPT}), \\
& d_{n}^{E} \simeq\left(0.65 \times 10^{-16}\right) \cdot \bar{\theta} \mathrm{e} \cdot \mathrm{cm}(\mathrm{LQCD}) .
\end{aligned}
$$

Using data on the nEDM [17] we extract the following upper limits for $\bar{\theta}$

$$
\bar{\theta}=4.4 \times 10^{-10}(\mathrm{ChPT}), \quad \bar{\theta}=4.7 \times 10^{-10}(\mathrm{LQCD}) .
$$

Using the measurement on the nEDM with the limit $\left|d_{n}^{E}\right|<1.6 \times 10^{-26} \mathrm{e} \cdot \mathrm{cm}$ [17] we deduce $\bar{\theta}=2.4 \times 10^{-10}$ (ChPT) and $\bar{\theta}=2.6 \times 10^{-10}$ (LQCD). This result should be compared to the bound on $\bar{\theta}$ directly obtained from the experimental constraint on nEDM with $\bar{\theta}<$ $1.1 \div 6 \cdot 10^{-10}[8,18,19]$. Our calculation shows ( this finding is independent of the assumption that CPV $\eta, \eta^{\prime}$-decays are generated by the same mechanism as the nEDM) that the very tight experimental limits on the nEDM exclude large contributions to $\eta\left(\eta^{\prime}\right) \rightarrow \pi \pi$ decays beyond that captured by the Peccei-Quinn mechanism [20]. The main difference with our calculation is that in the Peccei-Quinn mechanism the CPV $\eta\left(\eta^{\prime}\right) \pi \pi$ couplings are suppressed by $M_{\pi}^{2}$ in the chiral limit. We opted to relax this constraint but the effect of this assumption is marginal. Note that the fact that our two-loop result does not contain chiral divergences essentially means that chiral symmetry does not play a role in our scenario, consistent with the assumption that the couplings $f_{\eta \pi \pi}$ and $f_{\eta^{\prime} \pi \pi}$ may not be suppressed by the pion mass squared.

In conclusion, we studied limits of the QCD CP- violating parameter $\bar{\theta}$ and branchings of the CP- violating rare decays $\eta \rightarrow \pi \pi$ and $\eta^{\prime} \rightarrow \pi \pi$ using a phenomenological Lagrangian approach. We particularly took into account both minimal and nonminimal couplings of the nucleon to the external photon and the interaction of the external photon with charged pion fields. The nEDM was induced by the CP violating $\eta\left(\eta^{\prime}\right) \rightarrow \pi \pi$ couplings. Obtained results will be important for the planned experiments on rare $\eta$ and $\eta^{\prime}$ meson decays at JEF [21].

The work is supported by the Ministry of Education and Science of the Russian Federation, project No 3.9594.2017/8.9.

\section{References}

[1] T. Chupp, P. Fierlinger, M. Ramsey-Musolf and J. Singh, Rev. Mod. Phys. 91 (2019) 015001

[2] M. Pospelov and A. Ritz, Annals Phys. 318 (2005) 119

[3] T. Gutsche, A. N. Hiller Blin, S. Kovalenko, S. Kuleshov, V. E. Lyubovitskij, M. J. Vicente Vacas and A. Zhevlakov, Phys. Rev. D 95 (2017) 036022

[4] A. S. Zhevlakov, M. Gorchtein, A. N. Hiller Blin, T. Gutsche and V. E. Lyubovitskij, Phys. Rev. D 99 (2019) 031703

[5] A. S. Zhevlakov, T. Gutsche and V. E. Lyubovitskij, arXiv:1904.08154 [hep-ph]

[6] D. Diakonov and M. I. Eides, Sov. Phys. JETP 54 (1981) 232 [Zh. Eksp. Teor. Fiz. 81 (1981) 434]

[7] E. Witten, Nucl. Phys. B 156 (1979) 269

[8] R. J. Crewther, P. Di Vecchia, G. Veneziano and E. Witten, Phys. Lett. 88B (1979) 123, Phys. Lett. 91B (1980) 487(E)

[9] M. A. Shifman, A. I. Vainshtein and V. I. Zakharov, Nucl. Phys. B 166 (1980) 493

[10] R. Aaij et al. [LHCb Collaboration], Phys. Lett. B 764 (2017) 233 
[11] A. Faessler, T. Gutsche, V. E. Lyubovitskij and K. Pumsa-ard, Phys. Rev. D 73 (2006) 114021

[12] V. Lensky and V. Pascalutsa, Eur. Phys. J. C 65 (2010) 195

[13] S. Weinberg, Phys. Rev. 166 (1968) 1568.

[14] L. Tiator et al., Eur. Phys. J. A 54 (2018) 210

[15] J. Gasser and H. Leutwyler, Phys. Rept. 87 (1982) 77.

[16] M. Tanabashi et al. [Particle Data Group], Phys. Rev. D 98 (2018) 030001.

[17] B. Graner, Y. Chen, E. G. Lindahl and B. R. Heckel, Phys. Rev. Lett. 116 (2016) 161601, Phys. Rev. Lett. 119 (2017) 119901(E)

[18] M. Pospelov and A. Ritz, Phys. Rev. Lett. 83 (1999) 2526

[19] B. K. Sahoo, Phys. Rev. D 95 (2017) 013002

[20] R. D. Peccei and H. R. Quinn, Phys. Rev. Lett. 38 (1977) 1440.

[21] L. Gan, JPS Conf. Proc. 13 (2017) 020063. 